\title{
9. New ways and environments for using the CLES framework
}

Kristina Mikkonen (Research Unit of Nursing Science and Health Management, University of Oulu, Oulu, Finland), kristina.mikkonen@oulu.fi;

Olga Riklikiene (Faculty of Nursing, Lithuanian University of Health Sciences, Kaunas, Lithuania),riklikiene@gmail.com

Keywords: clinical learning environment, cultural and linguistic diversity, healthcare education, internationalism, supervision, medical students, student midwives, nursing, primary healthcare, specialized healthcare

Clinical Learning Environment and Supervision scale (CLES) has been used and validated in many countries (see Chapter 4). Most commonly, the validation studies have been carried out in a hospital environment and the samples have consisted of general nursing students. An interesting enquiry was raised concerning the validity and implications of the CLES framework in other fields of healthcare services and healthcare student groups including medical students. This chapter presents new ways and environments for implementing the CLES scale, with healthcare students in general, and international nursing students in particular. The examples come from healthcare and medical education. The section dealing with challenges relating to international students' clinical practicum also introduces a new scale Cultural and Linguistic Diversity scale (CALDs). This scale is developed for international nursing students and can be used together with the CLES+T scale.

\subsection{Healthcare and medical students}

In order to ensure the quality of the clinical learning environment and supervision of healthcare students, Clinical Learning Environment and Supervision scale (CLES) has been used and validated for various professional contexts. Three examples of original studies examining the use of the CLES scale include nursing students (Bos et al. 2012) and medical students (Öhman et al. 2016) in primary healthcare in Sweden; and midwifery students in primary and specialized healthcare in Lithuania (Kontrimaite 2017).

\section{Example from Sweden - nursing students in primary healthcare}

The investigation examined nursing and medical students' perspectives on the clinical learning environment in primary healthcare settings in Sweden (Bos 2014, Öhman et al. 2016). Students' perceptions on primary healthcare as a learning environment were measured using the CLES+T scale on nursing students (Bos et al. 2012) and CLES scale on medical students (Öhman et al. 2016). The purpose was to identify factors that promote a constructive) clinical learning environment in primary healthcare settings. This was the first time the scale was used and validated in a new learning environment. Table 9.1 presents the newly validated CLES+T scale for nursing students for primary healthcare settings.

The evaluation of CLES+T scale in the Swedish version (Johansson et al. 2010) for nursing students at primary healthcare demonstrated the scale as adequately relevant after the face and content validity analysis was performed by an expert panel of seven district nurses (Bos et al. 2012). The original items of the CLES+T scale (Saarikoski et al. 2008) were not modified for primary healthcare in the study. The experts evaluated each item in the CLES+T scale for their relevance in the Swedish primary healthcare context and for comprehensibility regarding all items. Construct 
validity with a confirmatory factor analysis was tested after data collection in a survey with 356 undergraduate nursing students (Bos et al. 2012). The CLES+T scale comprised of a five-factor model with 34 items. The results of the confirmatory factor analysis indicated that supervisory relationship was the most important factor. Supervisory relationship correlated strongly with pedagogical atmosphere and premises of nursing, moderately with the leadership style of Ward Manager and marginally with the role of the nurse teacher. The results confirmed good internal reliability of the CLES+T scale in investigating students' perceptions of the clinical learning environment and supervision in primary healthcare (Bos et al. 2012; Bos 2014).

The study highlighted that while different clinical settings make different demands on nursing students, each unique feature of that environment should be taken into account when designing learning programs and educational strategies. Moreover, it was evident that assessing supportive factors collectively rather than individually generates new information for the effective evaluation of the clinical learning environment of students (Bos et al. 2012).

\section{Example from Sweden- medical students in primary healthcare}

This validation of the CLES scale in its Swedish version (Johansson et al. 2010) was performed for medical students at the primary healthcare. The version was shown to be both adequately relevant and important for the context (Öhman et al. 2016). The original items of the CLES+T scale (Saarikoski et al. 2008) were modified specifically for primary healthcare. Connected with this, the sub-dimension Role of Nurse Teacher was removed. After the modification of the items of the scale, an expert panel of physicians evaluated face and content validity of the CLES scale. Construct validity with explorative factor analysis was tested in a survey with 394 medical students after the data collection. The CLES scale comprised of a four-factor model with 25 -items. The subdimensions of the newly validated CLES scale were further confirmed with a confirmatory factor analysis. Construct validity was established with outcomes closely comparable to the original CLES+T scale (Saarikoski et al. 2008). One item of the original scale loaded on a different subdimension, which was possibly caused by rephrasing of an original item in the study. Reliability and internal consistency of the newly validated CLES for medical students in primary healthcare settings was estimated as high. The scale was shown to be well applicable for measuring the clinical learning environment and supervision of medical students in primary healthcare (Öhman et al. 2016). Table 9.1 presents the newly validated CLES scale for medical students for primary healthcare settings. The results of the study made on the clinical learning environment and supervision of medical students in primary healthcare were not reported (Öhman et al. 2016).

\section{Example from Lithuania - midwifery students' clinical learning assessment}

The investigation was made on midwifery students' perspectives and their perceptions of the clinical learning environment in primary and specialized healthcare settings in Lithuania (Kontrimaite 2017). The Lithuanian version of the CLES scale, which had been previously validated for nursing students (Riklikiene and Nalivaikiene 2013), was used for midwifery students in the study. The original items of the CLES scale (Saarikoski 2002) were modified by changing the wording from mentor nurse to nurse/midwife. This double position was used due to the organization of clinical training for midwifery students in Lithuania. During different clinical training periods, the students practice in different hospital units, achieving learning outcomes that relate to general nursing practice and midwifery practice. In these situations both nurses and midwives serve as mentors, together with those nurse/midwifery ward managers who participate in organizing clinical training. All available students $(N=125)$ from the midwifery program at the university (55 students) (160 ECTS, 4 year degree program) and in college (70 students) (120 ECTS, 3 year degree 
program) participated in the survey just after they finished their clinical practicum period in primary or specialized healthcare settings. Reliability and internal consistency of CLES for midwifery students was estimated (see Table 9.2). The value of split half test (Spearman Brown correlation) was - 0.62 (Kontrimaite 2017).

The CLES scale created a possibility to conduct the first ever study in Lithuanian investigating the clinical training of midwifery students. This gave an opportunity to provide helpful information about the quality of clinical learning in the university hospital and other facilities (maternity, primary healthcare settings). The study revealed that midwifery students who were 22 or older evaluated their relationship with their supervisor during clinical learning as more positive than the younger students. Students at surgical and gynecological wards expressed greater satisfaction with their clinical practicum than students at any other ward. Also, students who were supervised under an individual mentorship model rated their satisfaction with clinical training as higher than those who had no supervisory relationship. Students in the final term of the midwifery program gave higher ratings for their relationship with their mentor compared with first year students. Similarly, graduates assessed individual supervision and feedback and equality in their supervisory relationship more positively than first year students. Also, midwifery students in their last study year preferred having a midwife as their mentor to any other health care professional at their clinical placement. When evaluating the pedagogical atmosphere on the ward, student midwives in their last study year more often felt, comfortable at the start of their shift, during meetings and when taking part in discussions, and appreciated staff interest in student supervision. Graduates, too, regarded the ward as a good learning environment. However, the results were significantly lower for first year student midwives. (Kontrimaite 2017).

\subsection{International nursing students}

An evaluation of the theoretical framework of the CLES+T scale demonstrated adequate relevance and importance relating to international students (Mikkonen et al. 2017a). With regards to international students' education, five areas in the CLES+T scale, the content of supervisory relationship, pedagogical atmosphere, premises of nursing on the ward, leadership style of the ward manager and role of the nurse teacher were complemented with additional five areas important specifically for them. These specific areas included: cultural diversity in the clinical learning, role of the student, orientation into the clinical placement, culturally diverse pedagogical atmosphere and linguistic diversity in the clinical learning environment (Mikkonen et al. 2017a).

The scales have been tested for face and content validity, and for construct validity with two explorative factor analyses on the CLES+T scale and the newly created Cultural and Linguistic Diversity scale (CALDs). The tests were made with 231 international students in a cross-sectional study (Mikkonen et al. 2017a). After psychometric testing, CLES+T scale changed from an original five-factor model into an eight-factor model with 34-items remaining. CALDs scale comprised of a five-factor model with 21-items. Reliability and internal consistency of the newly validated CLES+T scale and CALDs for international nursing students was estimated as satisfactory. The Cronbach's alpha for each factor varied between 0.77 and 0.97 (Mikkonen et al. 2017a). Table 9.3 presents the newly validated CLES+T scale and CALDs for international nursing.

The newly created CALDs adds sub-dimensions related to international nursing students' clinical learning environment and supervision to the CLES+T scale. The cultural and linguistic diversity in the clinical learning environment add to the original CLES+T scale, acknowledgment of the international student's cultural and linguistic diversity and receiving the international student positively by treating them like other students (Mikkonen et al. 2017a). Awareness of the other 
person's culture includes an acknowledgement of the other person's differing language skills. Skills in the native language were demonstrated as having a strong effect upon the students' success in learning and in feeling welcomed at the clinical practicum. Students with lower language skills perceived the pedagogical atmosphere of the ward as more negative than their peers at a more advanced level. The students with low language skills experienced more discrimination, social isolation and stress because of their diverse background. This also led them to have to prove their competence to others (Mikkonen et al. 2017b). The learning environment needs to be offered to international students at the same level of quality as for national students, and it needs to include multi-dimensional and meaningful learning situations.

Student supervision should be developed by giving pedagogical education on mentoring international students. The education should encompass the essential elements of cultural competence with an emphasis on pedagogical approaches, which facilitate students' learning and growth in professionalism (Mackay et al. 2012, Scheele et al. 2011, Thomposon 2012). These elements include planning and guiding the student's learning process, evaluating the learning outcomes, creating a safe environment to learn and to reflect while allowing students to verbalize their learning process and possible questions, and involving students in teamwork and professional decision making.

It has been shown that international students go through major challenges in the beginning of their clinical practicum and they need additional time to orientate into the clinical learning environment of a different culture (Sedgwick et al. 2014). For these reasons the sub-dimension Orientation into clinical placements was added in CALDs. Orientation was found to be essential for students in introducing them to the clinical culture, routines, timetables and to show the students what is expected of them by mentors and clinical staff (Mikkonen et al. 2016a). Orientation is an important aspect of supervision because it relates to the pedagogical approach of introducing students to a new learning environment and guiding the students through their learning process (Mikkonen et al. 2017a).

Due to reducing human resources in academia, the role of a nurse teacher has been limited to visiting the student's clinical practicum (Jokelainen 2013). International students saw the role of the nurse teacher as more essential than native students. Language proficiency of the students had a strong effect upon how essential role the nurse teacher plays in collaboration between the clinical practicum and higher education institutions. Students with low language skills in the native language required significantly more support by their nurse teachers (Mikkonen et al. 2017b). Collaboration between the clinical practicum and higher education institutions has been shown to play an essential role in the student's success in the clinical learning environment (Barnett et al. 2010). One of the responsibilities given to higher education institutions is to prepare international students in the local language so that they are ready to enter their clinical practicum. The mentors need to receive sufficient knowledge about the students' background, curriculum of their degree program, level of the set learning outcomes, evaluation process and clear guidance on how to deal with challenging situations. The role of the nurse teacher does not have to be limited to visits, but can include integrating versatile pedagogical approaches (reflection diaries, regular feedback) and guidance of students also from distance.

The role of the student was measured in a sub-dimension of CALDs. The result was that the role of the student was essential also for international students' success in learning. The students' perseverance, motivation and goal orientation has been shown to be an important aspect for success in international students' learning (Mikkonen et al. 2016b). Students' differing cultural backgrounds can lead to wrong interpretations on the basis of mentors' and other staff' non-verbal 
communication during the practicum. Culturally diverse behaviors may also be interpreted as a sign of not being willing to learn and as being unmotivated as students (Thompson 2012). Students need to be introduced to the concept of being active and independent learners with the distinctive meanings of the native country. Mentors would like students to be committed to their work schedules and show initiative in their learning (San Miguel and Rogan 2012). It has been previously emphasized that the students' own initiative in speaking the native language to patients and staff was received positively by their mentors (Koskinen and Tossavainen 2003). Encouraging students to be independent learners, with professional guidance from their mentors, can help international students to adapt to a new cultural reality and gain understanding of healthcare decisions made on the micro and macro levels of their host country.

\subsection{Conclusions and suggestions}

The validated instruments presented in the chapter may be implemented and used for improving healthcare and medical students' clinical learning environment and supervision. The validation of the instruments for multi-professional purposes in different learning environments involved minor adjustments of terminology relevant to each field of study. The terminology on clinical learning environments was exchanged from specialized clinical settings into primary healthcare settings. The measurement of the outcomes of medical education did not require to include the sub-dimension Role of Nurse Teacher. All versions of CLES+T and CLES discussed in the chapter presented sufficient validity in the studies. The reliability of CLES scale with midwifery students scored low, possibly because the sample used in the study was small.

Also, the framework of the CLES+T scale was confirmed as important for the clinical learning environment and supervision of international nursing students. However, there were additional aspects, which were missing in the CLES+T scale relating to international students learning' in the clinical environment. The CLES+T scale did not include cultural and linguistic diversity issues, which involve measuring the students' stressful experiences relating to their cultural backgrounds, language barriers relating to their learning outcomes, and their own role and initiative in learning when exposed to diversity and orientation in the clinical environment. An additional CALDs scale including all these aspects was created as a supplementary part to complement the theoretical framework of the CLES+T scale when measuring the outcomes of the international students' clinical learning environment and supervision.

Students require a safe learning environment in order to succeed in their clinical practicum. The collaboration systems between the clinical practicum and higher education institutions need to be improved. The suggested improvements include supporting the students' learning by clear clinical practicum procedures, evaluation systems, sufficient information provided about the students and sufficient guidance on how to deal with challenging situations. The CLES+T scale was shown to be an excellent instrument which may be used in healthcare and medical education in order to improve the clinical learning environment and supervision of students. The CLES+T scale, together with CALDs, can further improve international students' learning outcomes in clinical practicum and possibly be integrated into the orientation process of newly employed international staff in clinical environments.

\section{References}

Barnett T, Cross M, Shahwan-Akl L, Jacob E (2010) The evaluation of a successful collaborative education model to expand student clinical placements. Nurse Educ Pract 10: 71-21 
Bos E, Alinaghizadeh H, Saarikoski M, Kaila P (2012) Validating the 'clinical learning environment, supervision and nurse teacher' CLES+T instrument in primary healthcare settings using confirmatory factor analysis. J Clin Nurs 21 : $1785-1788$

Bos E (2014) A good learning environment for nursing students in primary health care. Theses for doctoral degree. Karolinska Institute, Stockholm, Sweden

Johansson U-B, Kaila P, Ahlner-Elmqvist M, Leksell J, Isoaho H, Saarikoski M (2010) Clinical learning environment, supervision and nurse teacher evaluation scale: psychometric evaluation of the Swedish version. J Adv Nurs 66: 2085-2093

Jokelainen M (2013) The elements of effective student nurse mentorship in placement learning environments. Systematic review and Finnish and British mentors' conceptions. Doctoral dissertation in health sciences. University of Eastern Finland, Publications of the University of Eastern Finland

Kontrimaite R (2017) Evaluation of clinical learning and teaching of midwifery students. Bachelor's theses, Lithuanian University of Health Sciences, Kaunas

Koskinen L, Tossavainen K (2003) Relationships with undergraduate nursing exchange students - a tutor perspective. J Adv Nurs 41(5): 499-508

Mackay B, Harding T, Jurlina L, Scobie N, Khan R (2012) Utilising the Hand Model to promote a culturally safe environment for international nursing students. Nurse Edu in Practic 12(2): 120-126

Mikkonen K, Elo S, Kuivila HM, Tuomikoski AM, Kääriäinen M (2016a) Culturally and linguistically diverse healthcare students' experiences of learning in a clinical environment: a systematic review of qualitative studies. Int J Nurs Stud 54: 173-187

Mikkonen K, Elo S, Tuomikoski AM, Kääriäinen M (2016b) Mentor experiences of international healthcare students' learning in a clinical environment: a systematic review. Nurse Educ Today 40: 87-94

Mikkonen K, Elo S, Miettunen J, Saarikoski M, Kääriäinen M (2017a) Development and testing of the CALDs and CLES+T scales for international nursing students' clinical learning environments. J Adv Nurs doi: $10.1111 /$ jan. 13268

Mikkonen K, Elo S, Miettunen J, Saarikoski M, Kääriäinen M (2017b) Clinical learning environment and supervision of international nursing students: a cross-sectional study. Nurse Educ Today 52: 73-80

Öhman E, Alinaghizadeh H, Kaila P, Hult H, Nilsson GH, Salminen H (2016) Adaptation and validation of the instrument Clinical Learning Environment and Supervision for medical students in primary health care. BMC Med Educ 16: 308

Riklikiene O, Nalivaikiene R (2013) Student nurses' assessment of pedagogical atmosphere on the ward during practical placement at a University Hospital in Lithuania. NERP 5:182-188

Saarikoski M (2002) Clinical Learning Environment and Supervision. Development and validation of the CLES evaluation scale. Doctoral dissertation. Annales Universitatis Turkuensis, University of Turku

Salamonson Y, Everett B, Koch J, Andrew S, Davidson PM (2008) English-language acculturation predicts academic performance in nursing students who speak English as a second language. Res Nurs Health 31(1): 86-94

San Miguel C, Rogan F (2012) Clinical expectations: what facilitators expect from ESL students on clinical placement. Nurse Educ Pract 12(2): 115-119

Scheele TH, Pruitt R, Johnson A, Xu Y (2011) What do we know about educating Asian ESL nursing students? A literature review. Nurse Educ Pract 32(4): 244

Sedgwick M, Oosterbroek T, Ponoma V (2014) "It all depends": how minority nursing students experience belonging during clinical experiences. Nurs Educ Perspect 35(2): 89-93 
Thompson CJ (2012) Improving the educational experience for international clinical nurse specialist students, part II. Clin Nurse Spec 11/12: 336-340 


\section{New ways and environments for using the CLES framework}

Table 9.1. CLES+T scale for nursing students and CLES scale for medical students in primary healthcare

\begin{tabular}{|c|c|c|c|}
\hline $\begin{array}{l}\text { CLES+T scale (34-items) for nursing } \\
\text { students }(n=356) \text { in primary healthcare } \\
(\text { Bos et al. 2012) }\end{array}$ & $\begin{array}{l}\text { Cron- } \\
\text { bach's } \\
\text { Alpha } \\
\end{array}$ & $\begin{array}{l}\text { CLES scale }(25 \text {-items }) \text { for medical } \\
\text { students }(n=394) \text { in primary healthcare } \\
\text { (Öhman et al. 2016) }\end{array}$ & $\begin{array}{l}\text { Cron- } \\
\text { bach's } \\
\text { Alpha } \\
\end{array}$ \\
\hline Factor 1- Supervisory relationship & 0.92 & Factor 1- Supervisory relationship & 0.91 \\
\hline Factor 2- Pedagogical atmosphere & 0.93 & $\begin{array}{l}\text { Factor 2- Pedagogical atmosphere at the } \\
\text { PHC centre }\end{array}$ & 0.92 \\
\hline Factor 3- Role of nurse teacher & 0.95 & $\begin{array}{l}\text { Factor 3- Leadership style of the manager } \\
\text { of the PHC centre }\end{array}$ & 0.95 \\
\hline Factor 4- Leadership style & 0.95 & Factor 4- Premises of patients & 0.95 \\
\hline Factor 5- Premises of nursing & 0.94 & & \\
\hline
\end{tabular}

Table 9.2. CLES scale for midwifery students in primary and specialized healthcare settings

CLES scale (25-items) for midwifery students in primary and specialized healthcare Cronbach's settings $(n=125)$ (Kotrimaite 2017) Alpha

\begin{tabular}{lc}
\hline Factor 1- Supervisory relationship & 0.73
\end{tabular}

$\begin{array}{ll}\text { Factor 2- Pedagogical atmosphere } & 0.65\end{array}$

Factor 3- Leadership style of the manager $\quad 0.65$

Factor 4- Premises of patients 0.68

Table 9.3. CLES+T scale and CALDs for international nursing students

\begin{tabular}{llll}
\hline $\begin{array}{l}\text { CLES+T scale (34-items) } \\
(\boldsymbol{n}=\mathbf{2 0 8}) \text { (Mikkonen et al. 2017a) }\end{array}$ & $\begin{array}{l}\text { Cron- } \\
\text { bach's } \\
\text { Alpha }\end{array}$ & $\begin{array}{l}\text { CALDs (21-items) } \\
(\boldsymbol{n}=\mathbf{2 1 4}) \text { (Mikkonen et al. 2017a) }\end{array}$ & $\begin{array}{l}\text { Cron- } \\
\text { bach's } \\
\text { Alpha }\end{array}$ \\
\hline Factor 1- Content of supervisory relationship & 0.97 & $\begin{array}{l}\text { Factor 1- Cultural diversity in } \\
\text { the clinical learning } \\
\text { environment }\end{array}$ & 0.85 \\
$\begin{array}{l}\text { Factor 2- Pedagogical atmosphere } \\
\text { Factor 3- Nursing care on the ward }\end{array}$ & 0.79 & $\begin{array}{l}\text { Factor 2- Role of the student } \\
\text { Factor 3- Orientation into the } \\
\text { clinical placement }\end{array}$ & 0.79 \\
$\begin{array}{l}\text { Factor 4- Role of the nurse teacher: } \\
\text { cooperation between } \\
\text { placement staff and nurse } \\
\text { teacher }\end{array}$ & 0.87 & $\begin{array}{l}\text { Factor 4- Culturally diverse } \\
\text { pedagogical atmosphere }\end{array}$ \\
$\begin{array}{l}\text { Factor 5- Role of the nurse teacher: } \\
\text { nurse teacher as enabling } \\
\text { the integration of theory } \\
\text { and practice }\end{array}$ & 0.90 & $\begin{array}{l}\text { Factor 5- Linguistic diversity in the } \\
\text { clinical learning } \\
\text { environment }\end{array}$ & 0.80 \\
$\begin{array}{l}\text { Factor 6- Leadership style of the } \\
\text { ward manager }\end{array}$ & & & 0.77 \\
$\begin{array}{l}\text { Factor 7- Role of the nurse teacher: } \\
\text { relationship } \\
\text { between student, mentor and } \\
\text { nurse teacher } \\
\text { Factor 8- Learning environment }\end{array}$ & 0.84 & & \\
\hline
\end{tabular}

